\title{
COMPARING THE DESIGN NEUROCOGNITION OF MECHANICAL ENGINEERS AND ARCHITECTS: A STUDY OF THE EFFECT OF DESIGNER'S DOMAIN
}

\author{
Vieira, Sonia Liliana da Silva (1); Gero, John S. (2); Delmoral, Jessica (1); Gattol, Valentin (3); \\ Fernandes, Carlos (4); Fernandes, António A. (5) \\ 1: INEGI Institute of Science and Innovation in Mechanical and Industrial Engineering, Porto, Portugal; \\ 2: Department of Computer Science and School of Architecture University of North Carolina at \\ Charlotte, United States; 3: AIT Austrian Institute of Technology, Vienna, Austria; 4: FMUP Faculty of \\ Medicine of the University of Porto, Portugal; 5: FEUP Faculty of Engineering, University of Porto, \\ Portugal
}

\begin{abstract}
New tools from neuroscience allow design researchers to explore design neurocognition. By taking the advantage of EEG's temporal resolution we give up spatial resolution to focus on the performance of time-related design tasks. This paper presents results from an experiment using EEG to measure brain activation to study mechanical engineers and architects to compare their design neurocognition. In this study, we adopted and extended the tasks described in a previous fMRI study of design neurocognition reported in the literature. The block experiment consists of a sequence of 3 tasks: problem solving, basic design and open design using a physical interface. The block is preceded by a familiarizing pre-task using the physical interface and then extended to a fourth task using free-hand sketching. Brainwaves were collected from both mechanical engineers and architects. Results comparing 36 mechanical engineers and architects while designing were produced. These results indicate design cognition differences between the two domains in task-related power between the problem-solving task and the design tasks, in temporal resolution and transformed power.
\end{abstract}

Keywords: Design cognition, Research methodologies and methods, Human behaviour in design, Design domains, Design neuroscience

Contact:

Vieira, Sonia Liliana da Silva

University of Porto

INEGI

Portugal

vieirasonia88@gmail.com

Cite this article: Vieira, S.L.S., Gero, J.S., Delmoral, J., Gattol, V., Fernandes, C., Fernandes, A.A. (2019) 'Comparing the Design Neurocognition of Mechanical Engineers and Architects: A Study of the Effect of Designer's Domain', in Proceedings of the 22nd International Conference on Engineering Design (ICED19), Delft, The Netherlands, 5-8 August 2019. DOI:10.1017/dsi.2019.191 


\section{INTRODUCTION}

One of the questions that design research has studied is whether designing is domain specific or has general characteristics that are independent of any domain of application (Goel and Pirolli, 1992). There is some consensus on the notion that although the foundations of design are generic, the practice of design differs in different domains through an increase or decrease in the various activities that make up the foundations of designing (Gero and Kannengiesser, 2014; Visser, 2009). How different domains practice design is of particular interest as design thinking has become an approach that claims to transcend domains. In design education this is of interest as it affects whether it is possible to teach design as a generic activity that is then supplemented by domain specific practice.

For the last 25 years design cognition has increasingly been studied using the protocol analysis method (Cross, Christiaans, and Dorst, 1996; Ericsson and Simon, 1993; Kan and Gero, 2017; Van-Someren, Barnard, and Sandberg, 1994). Protocol analysis-based studies have yielded a range of rich insights into design as a process. These insights include the design cognition of domains (Akin, 2001; Cross and Roozenburg, 1992; Schön, 1988).

Recently new non-invasive tools have provided access to brain behaviour through objective measurements of various aspects of brain neurophysiology producing the field of neurocognition (Ward, 2015). This has opened the way for the first steps in understanding design neurocognition - the neurocognition of designing. Functional magnetic resonance imaging (fMRI)-based design neurocognition studies are at the exploratory stage with one well-controlled experiment published (Alexiou et al., 2009) and others that focus on design related behaviours rather than the acts of designing (Goucher-Lambert, Moss and Cagan, 2017). fMRI has very high spatial resolution which helps in narrowing down to very specific brain regions that are activated during any task, but it has low temporal resolution. Designing is a temporal activity. By taking the advantage of the electroencephalography (EEG) method's high temporal resolution, we can focus on the investigation of time-related design tasks. Design neurocognition EEG-based studies are emerging at an exploratory stage with a few reported domain-specific studies on engineering design, architecture and industrial design. Results from controlled experiments identify the relationship between design problem statements and cognitive behaviors during conceptual design (Liu et al., 2018), visual attention and association in expert designers (Liang et al., 2018), neurophysiological EEG signals to study effort, fatigue and concentration in conceptual design (Nguyen et al., 2016), EEG bands associated with the design activities of problem solving and evaluation (Liu et al., 2016), neurophysiological correlates of embodiment and motivational factors during the perception of virtual architecture (Vecchiato et al., 2015). However, time-related neural responses during problem-solving compared to design tasks across domains are as yet unknown. EEG's high temporal resolution during cognition can help elucidate the stages of the recognition of tasks while provide the temporal basis for information processing (Hinterberger et al., 2014). Averaging the measurements yields a measure of the EEG voltages that are consistently related to the sensory, perceptual and decision-making processes (Dickter and Kieffaber, 2014). Results from neurosciencebased design studies show consistent higher activation of the dorsolateral prefrontal cortex of design tasks and ill-structured problems (Alexiou et al., 2009), and insight (Kounios and Beeman 2014, Beeman et al., 2004), recruiting a more extensive network of brain areas than problem solving (Alexiou et al., 2009, Kounios and Beeman 2014). Neuroimaging studies are more advanced in creative cognition (Martindale, 1999; Vartanian and Goel, 2005), and visual creativity and the arts (Pidgeon et al., 2016), although no consensus has been found due to the different nature of the tasks across studies.

This study reported in this paper is part of an ongoing research to elucidate design neurocognition, using it to distinguish design from problem-solving and to determine differences in brain behaviour when design is carried out in different domains. The research is based on the analysis of participants' brain waves using an EEG headset in the context of performing problem solving and design tasks in an experimental environment. The objectives of the study reported here are to:

- investigate the use of the EEG technique to distinguish design from problem-solving.

- identify neurocognitive similarities and differences across design domains.

We postulate the following three hypotheses:

Hypothesis 1. Design neurocognition of mechanical engineers and architects are different.

Hypothesis 2. Neurocognitive temporal distributions of activations between mechanical engineers and architects are significantly different. 
Hypothesis 3. Neurocognitive temporal distributions of activations of mechanical engineers and architects are non-uniform.

\section{METHODS}

We have adopted and replicated the tasks described in Alexiou et al. (2009), expanding their results into the temporal domain using EEG's high temporal resolution. We extend the experiment to a further task. The set of tasks is preceded by a pre-task so that the participants can familiarize themselves with the physical interface. The original tasks are followed by a third layout task and a fourth task based on free-hand sketching. The replication of the experiment tasks of Alexiou et al. (2009) with EEG brain wave data is supported with the analysis of data from video and audio recording and observation for later protocol analysis.

\subsection{Experiment setup}

A physical interface for individual task performance was built based on magnetic material for easy handling. A pre-task was designed so that participants can familiarize themselves with the use of the headset, maneuvering the magnetic pieces and prevent them from getting fixated in Task. 1. This pretask also has the goal to familiarize participants with postural and facial movements and allows the researcher to correct and fine tune so that the recording have low interference. The Mikado game was given to the participants to play in the breaks between tasks as they have to accurately pick each piece of the game this action helps them with the physical interface of the magnetic and movable pieces during the tasks. The block experiment consists of a sequence of 3 tasks: problem-solving, basic design and open design, Table 1 and Figure 1. We have matched Tasks 1 and 2 with the problemsolving and design tasks from Alexiou et al. ( 2009) as closely as possible in terms of number of constraints, stimuli, number of instructions so that the cognitive effort to understand them is similar. Task 3 provides an enlargement of the problem and the solution space and the opportunity of evaluating and reformulating the design solutions. In Task 4, free-hand sketching, the participants are asked to propose and represent an outline design. Each participant is given two sheets of paper (A3 size) and three instruments, a pencil, graphite and a pen. The participant is asked to: propose and represent an outline design for a future personal entertainment system. Here, the purpose is to observe differences in participants' brain activation due to the nature of the tasks.

Table 1. Description of the problem-solving, basic design and open design tasks.

Task 1 Problem-solving Task 2 Basic design

Task 3 Open design

In Task 1 the design of a set of furniture is available and three conditions are given as requirements. The task consists of placing the magnetic pieces inside a given area of a room with a door, a window and a balcony.

In Task 2 the same design set of furniture is available, and three requests are made. The basic design task consists of placing the furniture inside a given room area according to each participant notions of functional and comfortable using at least three pieces.
In Task 3 the same design available is complemented with a second board of movable pieces that comprise all the fixed elements of the previous tasks, namely, the walls, the door, the window and the balcony. The participant is told to arrange a space.

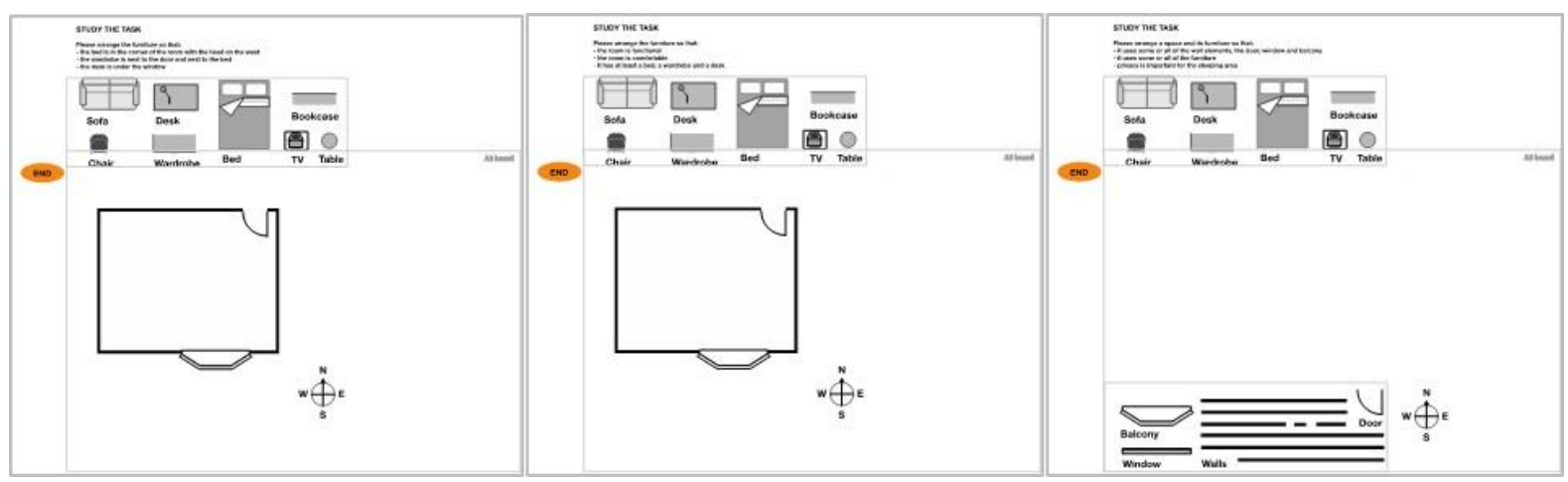

Figure 1. Depiction of the problem-solving Task 1, basic-design Task 2 and open design Task 3. 
For Tasks 1 and 2 (Alexiou et al., 2009), the movable pieces were placed at the top of a vertical magnetic board to prevent signal noise due to eye and head movements as tested in the physical magnetic board. The EEG activity is recorded using a portable 14-channel system Emotiv Epoc+. Electrodes are arranged according to the 10-10 I.S, Figure 2. The subjects performed the tasks on a physical magnetic board, with two video cameras for capturing the participant face and activity and an audio recorder.
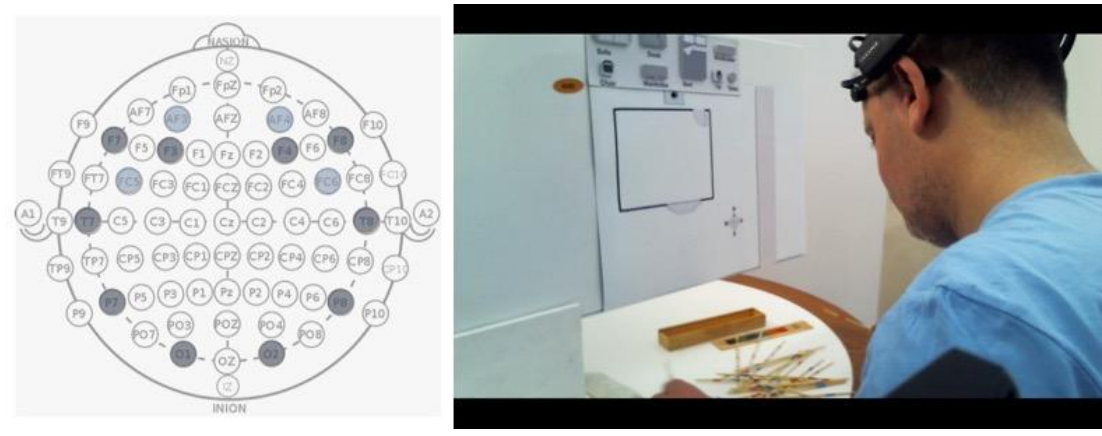

Figure 2. Emotiv Epoc+ electrodes arrangement (10-10 I.S.) and experiment setup using the headset.

All the data captures were streamed using Panopto software (https://www.panopto.com/) that also allows for direct screen capture, Figure 3. One researcher is present in each experiment episode for recordings and instructing the participant. A period of 10 minutes for setting up and a few minutes for a short introduction are necessary for informing each participant, reading and signing of the consent agreement and discussing the experiment. The researcher positions the participant at the desk and checks metallic accessories for electromagnetic interference, as well as setting the room temperature.

The researcher asks each participant's attention for neck movements, blinking, muscle contractions as well, rotating the head, horizontal eye movements, pressing the lips and teeth, and silly faces in particular during the tasks, as these affect the signal capture. Electromagnetic interference of the room was checked for frequencies below $60 \mathrm{~Hz}$. The researcher follows a script to conduct the experiment so that each participant gets the same information and stimuli. Before each task, participants were asked to start by reading the text which took an average of $10 \mathrm{~s}$ of reading period. Then the subjects performed the sequence of five tasks previously described with breaks in between where they play Mikado. The experiments took a total time between 34 to 66 minutes. The experiments took place between March and July of 2017, and June and September of 2018 in a room with the necessary conditions for the experiment, such as natural lighting from above sufficient for performing experiments between 9:00 and 15:00 and no electromagnetic interference. An open question interview took place in two distinct moments, at the end of Task 3 and 4.

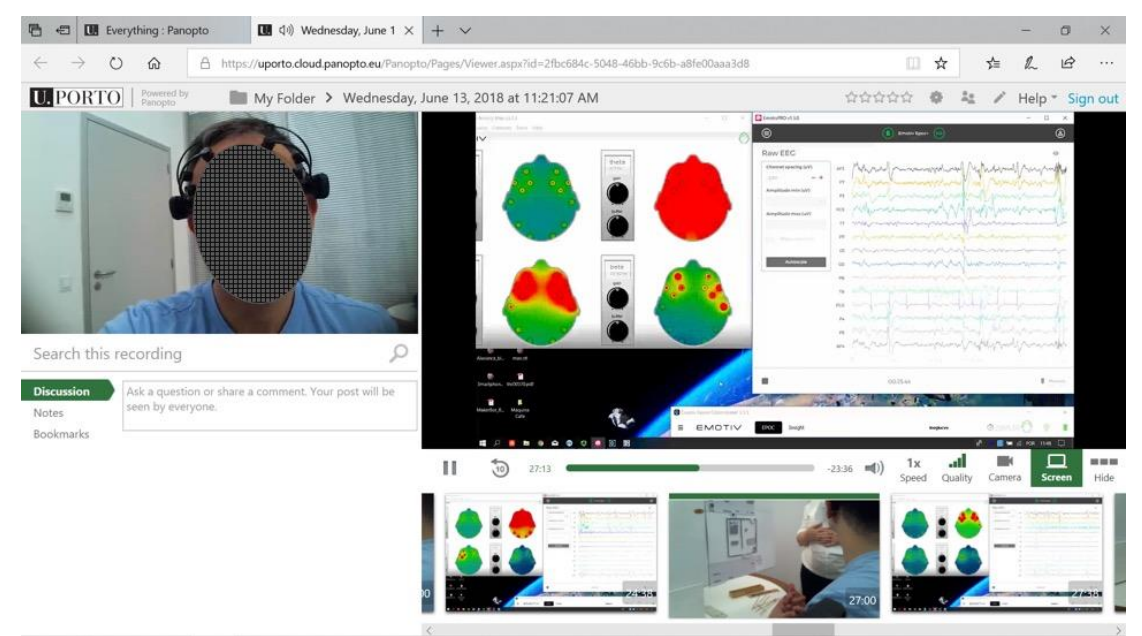

Figure 3. Screen capture depicting audio, video and screen captures streaming in Panopto.

Each participant was reminded to use the bathroom and spit out any gum before the start of the experiment. The researcher sits each participant at the desk, asks him/her to untie hair and remove earrings and other metallic accessories of electromagnetic interference, check if they are using contact lenses as these may cause to much blinking and interfere with data collection. Time was given to the 
participants, in particular in Tasks 3 and 4 so they could find a satisfactory solution. In the short interviews conducted at the end of Tasks 3 and 4 the researcher asks participants four open questions. The oral responses to the open questions were recorded and transcribed for analysis. A 5 Factor Personality Test given to each participant after the experiment. The 100 item questionnaire was available online which participants accessed through a link sent by email or responded in place. Results of the interviews and the personality tests will be reported elsewhere.

\subsection{Participants}

We invited mechanical engineers and architects demographically having the same characteristics (language, culture, background) to the experiment for exploratory comparison. Information on subjects' demographics was gathered beforehand. In this study we describe the analysis of 36 individuals. Results depicted are based on 18 architects aged $28-51(\mathrm{M}=38.9, \mathrm{SD}=5.3)$, and 18 mechanical engineers, aged $25-40(M=28.4, S D=3.7)$. The sample included 17 women $(M=32.5$, $\mathrm{SD}=5.0)$ and 19 men $(\mathrm{M}=34.7, \mathrm{SD}=8.3)$ all right-handed. This study was approved by the local ethics committee of the University of Porto.

\subsection{EEG data processing}

In this research we adopt the techniques developed for the removal of muscle artifacts from EEG recordings. The blind source separation (BSS) technique based on canonical correlation analysis was proposed to separate tonic muscle artifacts from continuous EEG recordings during epileptic events (De Clercq et al., 2006; Vergult et al., 2007). The same algorithm was adapted to remove the short EMG bursts due to articulation of spoken language, attenuating the muscle contamination on the EEG recordings and providing several research communities a tool to investigate the brain processes during language production (Vos et al., 2010). Thus, for the present study the results are based on data subjected to the blind source separation (BSS) technique for signal processing.

The EEG signals were recorded with electrodes placed according to 10-10 I.S, $256 \mathrm{~Hz}$ sampling rate, low cutoff $0.1 \mathrm{~Hz}$, high cutoff $50 \mathrm{~Hz}$. Data processing includes the removal of DC offset with the IIR procedure, and the previously mentioned blind source separation technique (BSS). Data analysis then proceeds with total and band power values, in the time-course of unfolding cognitive events, on individual and aggregate levels, in MatLab and open source software, based on transformed power (Pow) and task related power (TRP) calculations.

\subsection{EEG data analysis}

A total of 52 experiments were conducted, 26 per domain. Due to EEG recording issues four experiments were initially excluded. The analysis then proceeded based on the EEG data recorded and processed for each of the 48 remaining experiments, and each of the 14 electrodes used for averaging, for each of the tasks. For the analysis of the transformed power (Pow) across tasks per participant a ztransform was conducted to determine outliers. The criteria for excluding participants were based on the evidence of 6 our more threshold $\mathrm{z}$-score values above 1.96 or below -1.96 and individual measurements above 2.81 or under -2.81 . This resulted in a further 12 experiments being excluded leaving 36, circumstantially 18 for each domain.

We focus on the overall activation per channel, per task, per participant and domain as the study aim is to know how far results for problem solving and designing discriminate across the tasks. The taskrelated power (TRP) is typically calculated taking the resting state as the reference period per individual. We analyzed the EEG recordings of the resting periods prior to the experiment of a few participants and their results vary considerably, some participants show signals that can be associated with the state of being nervous (Liu et al., 2016) and expectant and their cognitive effort and activity is unknown. As the focus of the present study is to know how far designing can be distinguished from problem-solving we take the problem-solving Task 1 as the reference period for the TRP calculations. Thus, for each of the 14 electrodes, the following formula was applied taking the mean of the corresponding electrode $i$, in Task 1 as the reference period. By subtracting the log-transformed power of the reference period (Powi, reference) from the activation period (Powi, activation) for each trial $\mathrm{j}$ (each one of the five tasks per participant), separately, according to the formula:

$$
\mathrm{TRPi}=\log (\text { Powi, activation) } \mathrm{j}-\log (\text { Powi, reference }) \mathrm{j}
$$


By doing this, the negative values indicate a decrease of task-related power from the reference (problem-solving Task 1) for the activation period, while positive values express a power increase (Pfurtscheller and Lopes da Silva, 1999). TRP scores were quantified for total power and temporal analysis was initially carried out by dividing each experiment session into halves per task across domains. By power and activation, we refer to brain wave amplitude.

\section{ANALYSIS AND RESULTS}

Preliminary results of total task-related power (TRP) across the 36 participants, Figure 4, indicate that the tasks can potentially be distinguished from each other using the TRP values.

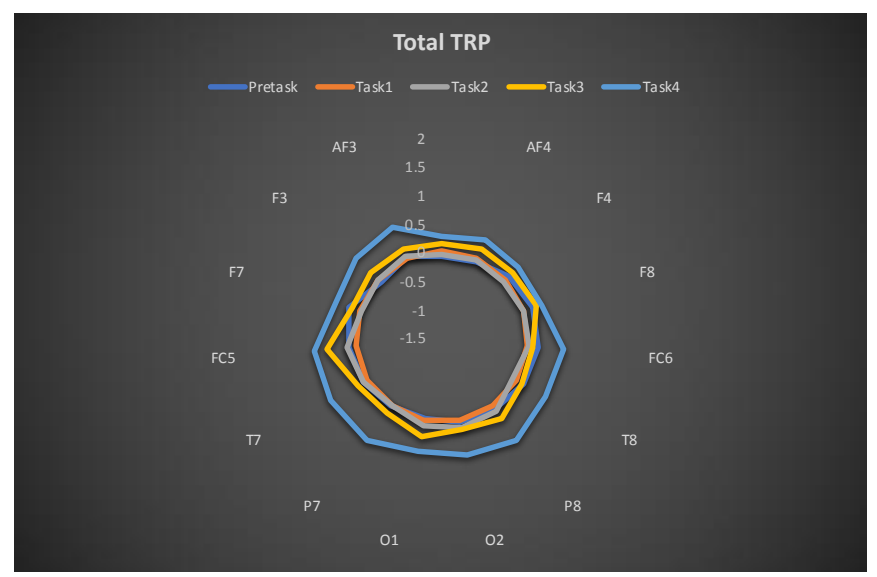

Figure 4. Task related power (TRP) for the 14 electrodes by taking problem solving Task 1 as the reference period across the 36 participants and the five tasks.

\subsection{Task-related power of mechanical engineers and architects}

The analysis of task-related power (TRP) allowed a preliminary comparison of differences between mechanical engineers and architects. Results between the two sets of tasks for the mechanical engineers and architects are depicted in Figure 5.

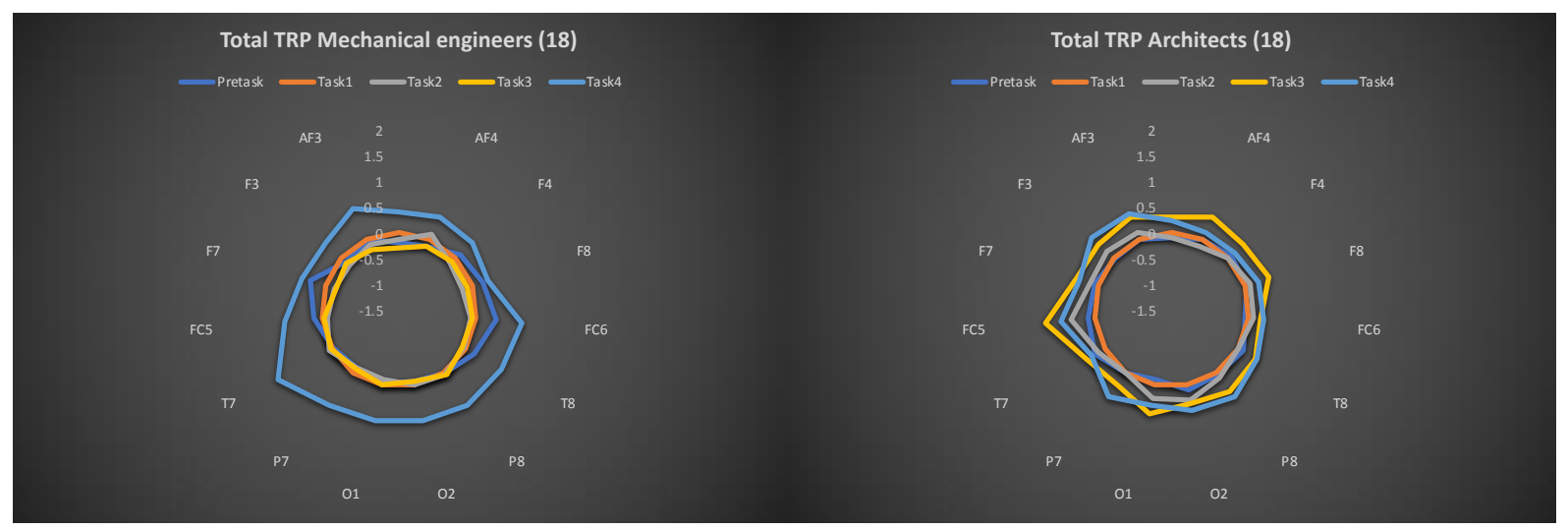

Figure 5. Task-related power (TRP) of the five tasks for the mechanical engineers and architects.

To compare the TRP of mechanical engineers and architects, we performed an analysis by running a $2 \times 4 \times 2 \times 7$ mixed repeated-measurement ANOVA, with the between-subjects factor domain and the within-subject factors task, hemisphere and electrode. From the analysis of the 36 participants (18 mechanical engineers and 18 architects) we found a significant main effect of task, $F(2.17$, 73.67) $=3.94, \mathrm{p}<0.02, \eta 2$ partial $=.10$ (corrected for Greenhouse-Geisser estimates of sphericity, $\varepsilon=$ .72). None of the other factors showed a significant main effect. Further, the ANOVA revealed a marginally significant interaction effect between the factors task and domain, $F(3,34)=2.27, p<0.09$, $\eta 2$ partial $=.06$; as well as a marginally significant interaction effect between the factors hemisphere and domain, $\mathrm{F}(1,34)=2.16, \mathrm{p}=0.15, \eta 2 \mathrm{partial}=.06$. No other two-way interactions were found to be significant or close to significant at this level of analysis. 
In addition, we conducted pairwise comparisons to check for differences between mechanical engineers and architects comparing the 7 electrodes per hemisphere and task. Below we report both on significant $(\mathrm{p} \leq 0.05)$ and close to significant $(\mathrm{p} \leq 0.15)$ pairwise comparisons. The following comparisons were marginally significant or significant. For Task 2, electrodes F3(p=0.13), FC5 $(\mathrm{p}=0.14)$ and $\mathrm{O} 1(\mathrm{p}=0.15)$ in the left hemisphere all electrode positions were marginally significant. For Task 3, electrodes $\mathrm{F} 4(\mathrm{p}=0.02), \mathrm{T} 8(\mathrm{p}=0.03)$ and $\mathrm{O} 2(\mathrm{p}=0.03)$ significant and $\mathrm{AF} 4(\mathrm{p}=0.15)$ and $\mathrm{P} 8(\mathrm{p}=0.06)$ marginally significant in the right hemisphere, and in the left hemisphere FC5 $(\mathrm{p}<.01)$ significant and $\mathrm{AF} 3(\mathrm{p}=0.06), \mathrm{F} 3(\mathrm{p}=.06), \mathrm{F} 7(\mathrm{p}=0.12), \mathrm{P} 7(\mathrm{p}=0.10)$ and $\mathrm{O} 1(\mathrm{p}=0.07)$ marginally significant. For Task 4 , electrodes FC6 $(p=0.11)$ in the right hemisphere and electrodes $\mathrm{T} 7(\mathrm{p}=0.14)$, in the left hemisphere, marginally significant. Some deactivations per group will be analyzed and interpreted in further studies. The indicators of significant and close to significant differences of some electrodes/regions and hemisphere across tasks are shown in Figures 6.

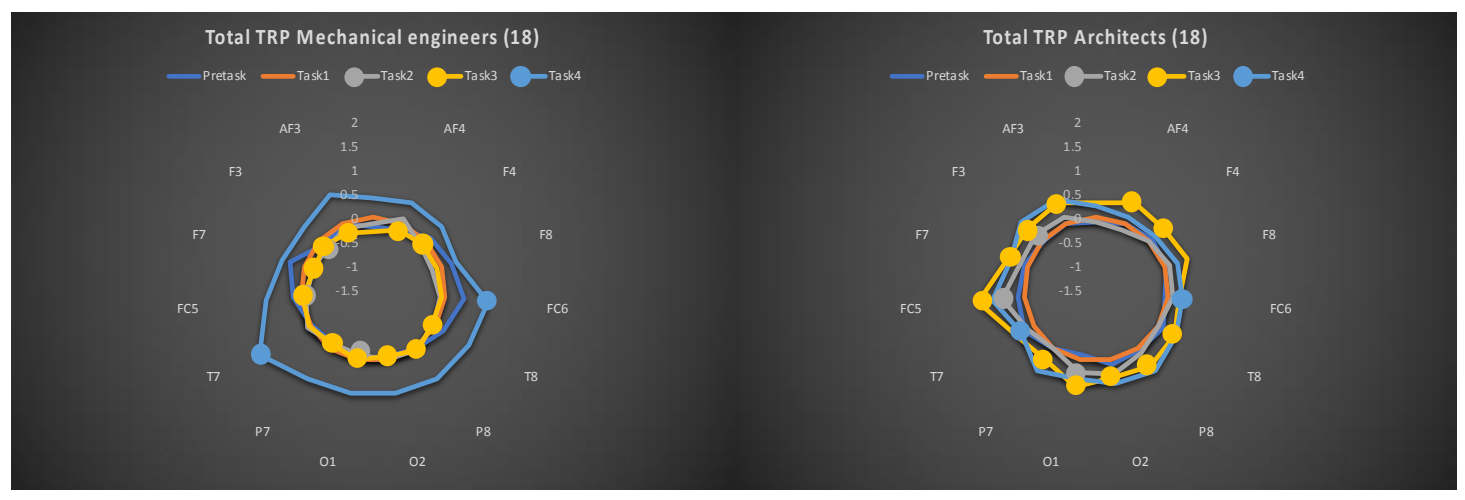

Figure 6. Preliminary indicators of significant or close to significant differences of electrodes/regions and hemisphere across tasks.

\subsection{Preliminary temporal analysis}

The transformed power (Pow) was calculated for the first and second halves of each task. Preliminary results across the 36 participants, per domain are depicted in Figure 7.
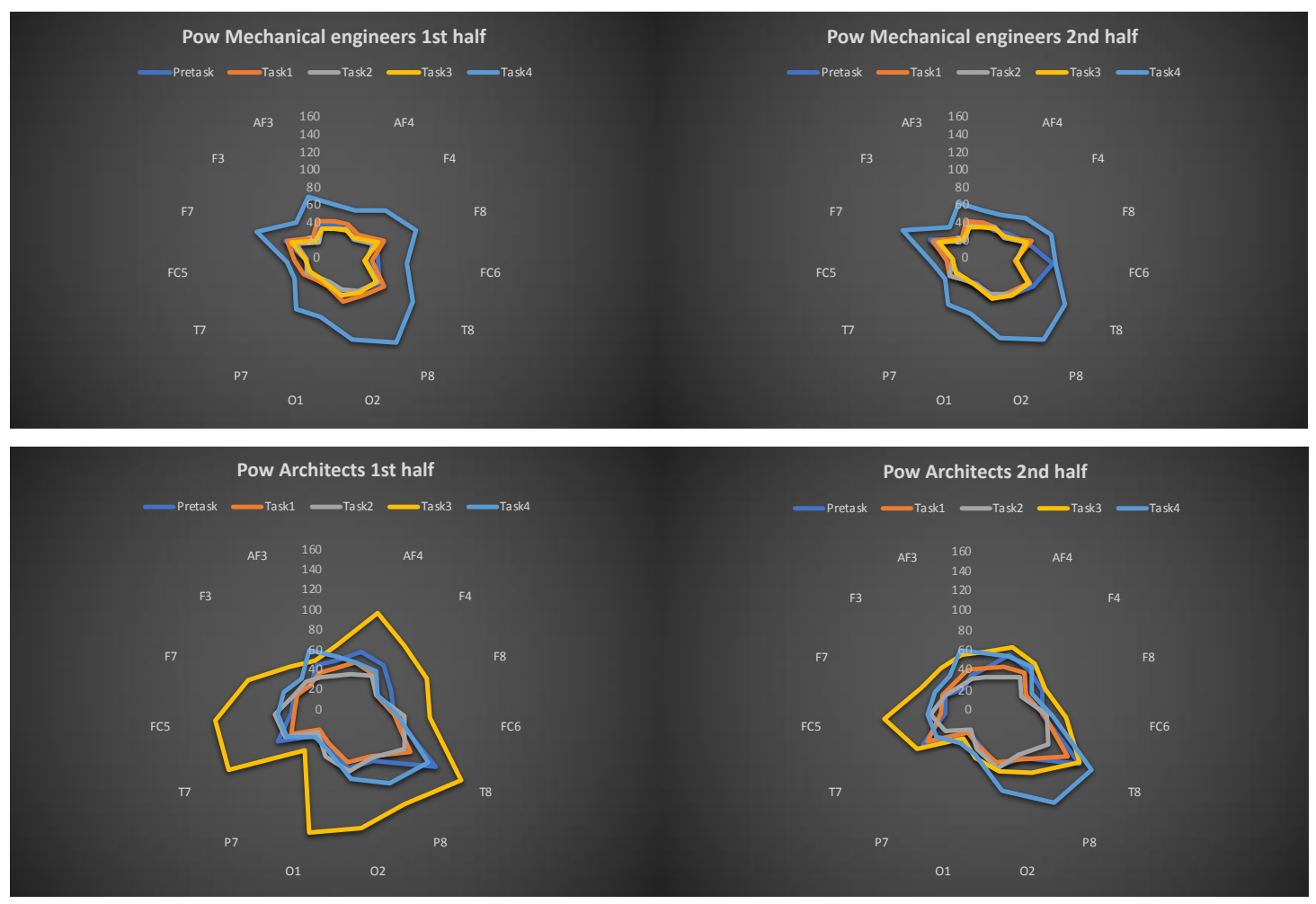

Figure 7. Transformed power (Pow) across mechanical engineers, architects and tasks first and second halves. 
The task related power (TRP), taking Task 1 as the reference period, was calculated for the first and second halves of each task. Preliminary results of TRP scores for the halves across the 36 participants, per domain, are depicted in Figure 8.
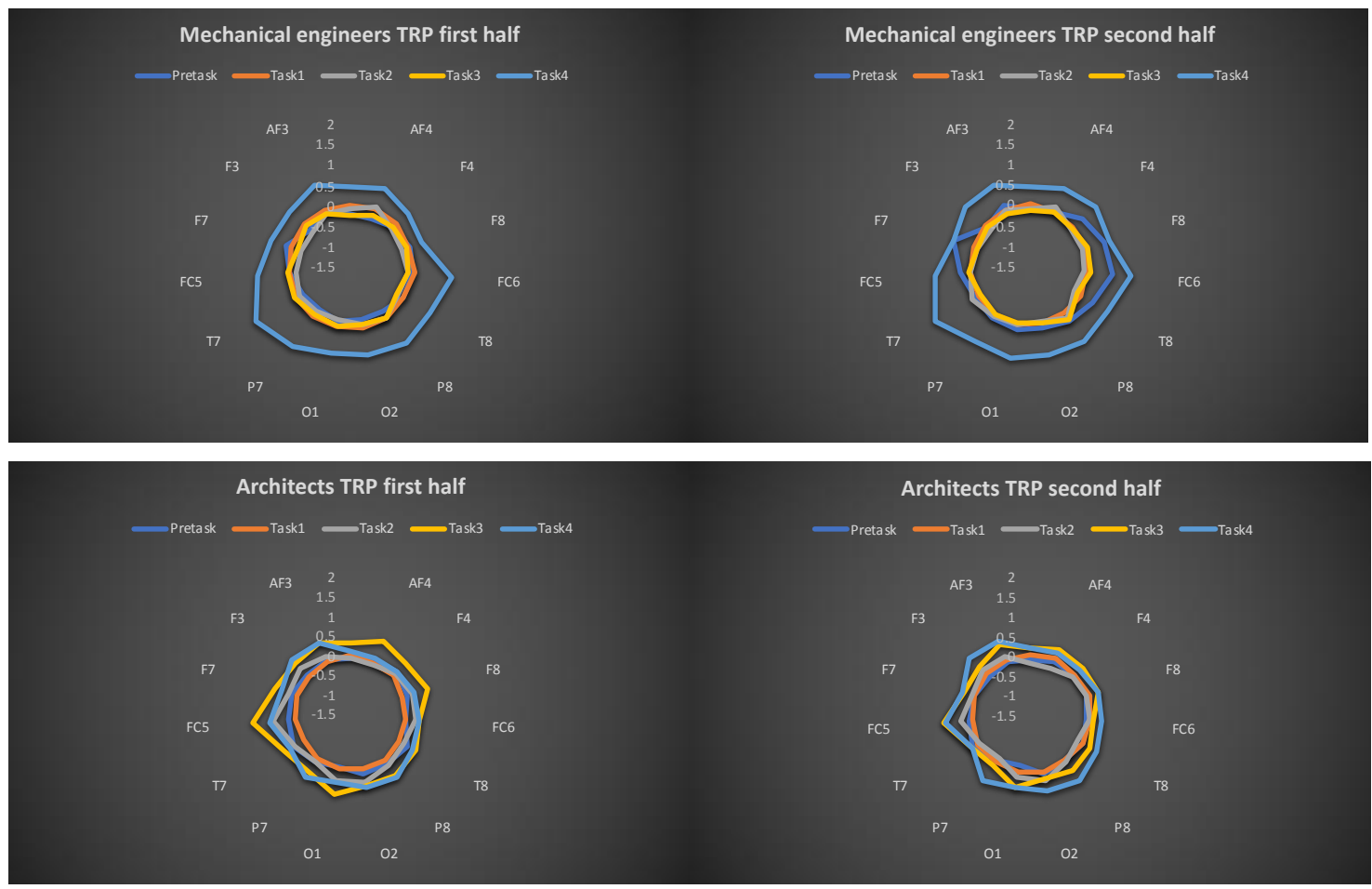

Figure 8. Task related power (TRP) across mechanical engineers and architects and tasks first and second halves.

To compare the TRP scores for the halves of mechanical engineers and architects, we performed an analysis by running a $2 \times 4 \times 2 \times 7 \times 2$ mixed repeated-measurement ANOVA, with the between-subjects factor domain and the within-subject factors task, hemisphere, electrodes and half. From the analysis of the 36 participants (18 mechanical engineers and 18 architects) we found a significant main effect of task, $\mathrm{F}(2.15,73.19)=4.77, \mathrm{p}=0.01, \eta 2$ partial $=.12$ (corrected for Greenhouse-Geisser estimates of sphericity, $\varepsilon=.72$ ). None of the other factors showed a significant main effect. Further, the ANOVA revealed a marginally significant interaction effect between the factors task and domain, $\mathrm{F}(3$, $34)=1.94, p<0.13, \eta 2$ partial $=.05$. No other two-way interactions were found.

In addition, we conducted pairwise comparisons to check for differences between mechanical engineers and architects comparing the 7 electrodes per hemisphere, task and half. Below we report both on significant $(\mathrm{p} \leq .005)$ and close to significant $(\mathrm{p} \leq 0.15)$ pairwise comparisons. The following comparisons were marginally significant or significant: for the Pretask, second half, electrode FC6 $(\mathrm{p}=0.12)$ marginally significant in the right hemisphere. For Task 2 , first half, electrodes $\mathrm{F} 3(\mathrm{p}=0.09)$, FC5 $(p=0.07)$ and $\mathrm{O} 1(\mathrm{p}=0.08)$ marginally significant in the left hemisphere and in the second half, electrode $\mathrm{O} 2(\mathrm{p}=0.14)$ marginally significant in the right hemisphere. For Task 3 , first half, electrodes $\mathrm{F} 4(\mathrm{p}=0.05), \mathrm{T} 8(\mathrm{p}=0.03)$ and $\mathrm{P} 8(\mathrm{p}=0.04)$, significant and $\mathrm{AF} 4(\mathrm{p}=0.09), \mathrm{F} 8(\mathrm{p}=0.14)$ and $\mathrm{O} 2(\mathrm{p}=0.07)$ marginally significant in the right hemisphere and in the left hemisphere FC5 $(p<0.01)$ and $\mathrm{AF} 3(\mathrm{p}=0.05)$, significant and $\mathrm{F} 3(\mathrm{p}=0.12), \mathrm{F} 7(\mathrm{p}=0.08)$ and $\mathrm{O} 1(\mathrm{p} 0=.15)$ marginally significant. For Task 3, second half, electrode $\mathrm{F} 4(\mathrm{p}=0.03)$, significant and $\mathrm{T} 8(\mathrm{p}=0.12)$ and $\mathrm{O} 2(\mathrm{p}=0.13)$ marginally significant in the right hemisphere and in the left hemisphere FC5 ( $\mathrm{p}=0.04)$, significant and $\mathrm{AF} 3(\mathrm{p}=0.15)$ and $\mathrm{O} 1(\mathrm{p}=0.11)$ marginally significant. For Task 4, first half, electrode FC6 $(\mathrm{p}=0.12)$, marginally significant in the right hemisphere and in the second half, electrode $\operatorname{FC6}(\mathrm{p}=0.12)$ marginally significant in the right hemisphere and $\mathrm{T} 7(\mathrm{p}=0.12)$, marginally significant in the left hemisphere. There was no adjustment for multiple comparisons. 


\section{DISCUSSION AND CONCLUSION}

Results from this study demonstrate that it is possible to address the overall objectives of this research: investigate the use of the EEG technique to distinguish design from problem-solving and identify similarities and cognitive differences across the design domains. The results of this preliminary analysis of the EEG data of the 36 participants show significant differences in the design neurocognition of mechanical engineers and architects and provide initial support for hypothesis 1: the design neurocognition of mechanical engineers and architects are different, particularly in Task 3 across domains and hemispheres. The layout Task 3 involves the enlargement of the problem and the solution spaces as well as the opportunity of evaluating and reformulating the previous design solutions. Architects show higher transformed power (Pow) and more distinct TRP differences from the problem-solving task than do mechanical engineers. The neurocognitive temporal distributions of activations between mechanical engineers and architects are significantly different providing initial support for hypothesis 2: the neurocognitive temporal distributions of activations between mechanical engineers and architects are significantly different, as mechanical engineers show more cognitive effort in the electrodes of the left prefrontal cortex in the problem-solving and design tasks, and architects show more cognitive effort in the electrodes of the temporal and occipital cortex of the right hemisphere. The neurocognitive temporal distributions of activations between mechanical engineers and architects are also significantly different providing initial support for hypothesis 3: the neurocognitive temporal distributions of activations of mechanical engineers and architects are nonuniform, as mechanical engineers show little variation in the Pow and TRP between the problemsolving and design tasks, first and second halves while architects show higher variation particularly in the problem-solving task and Task 3. Mechanical engineers show higher Pow and TRP difference from the problem-solving task in the sketching task. On a qualitative level the current study shows evidence of a distinct characteristic of increased Pow and TRP of design tasks from the reference problem solving task across mechanical engineers and architects.

Further studies will focus on band waves filtering, detailed analysis addressing temporal changes in neurocognition over time while designing and think-aloud protocol collected while measuring EEG responses. The removal of effects due to speech will allow the temporal matching of design cognition with neurophysiology, opening up a new research direction for neurocognitive research in design studies.

\section{ACKNOWLEDGMENTS}

This research was supported by the Unity of Conceptual and Experimental Validation of INEGIFEUP. We thankfully acknowledge the consultancy of the neurophysiologist Dílio Alves of the local hospital, Prof. João Paulo Cunha of INESC-Feup brain research group and Prof. Manoel Silva for the discernments on magnetism. The research is funded by the Portuguese Foundation for Science and Technology, grant number SFRH/BPD/104281/2014.

\section{REFERENCES}

Alexiou, K, Zamenopoulos, T, Johnson, J.H. and Gilbert S.J (2009), "Exploring the neurological basis of design cognition using brain imaging: some preliminary results", Design Studies, Vol. 30 No. 6, pp. 623-647.

Akin, O. (2001), "Variants in design cognition. In, Design Knowing and Learning: Cognition in Design Education", Editors, Eastman, C. Newstetter, W. and McCracken, M. Elsevier Science.

Beeman M., Bowden E., Haberman J., Frymiare J. and Arambel-Liu S. (2004), "Neural Activity When People Solve Verbal Problems with Insight," PLoS Biol Vol. 2 No. 4, p. e97. https://doi.org/10.1371/journal.pbio.0020097.

Cross, N., Christiaans, H. and Dorst, K. (1996), “Analysing Design Activity”, Wiley.

Cross, N. and Roozenburg, N. (1992), "Modelling the Design Process in Engineering and in Architecture", Journal of Engineering Design, Vol. 3 No. 4, pp. 325-337.

De Clercq, W., Vergult, A., Vanrumste, B., Van Paesschen, W. and Van Huffel, S. (2006), "Canonical correlation analysis applied to remove muscle artifacts from the electroencephalogram", IEEE Transactions on Biomedical Engineering, Vol. 53, pp. 2583-2587.

Dickter, C. and Kieffaber, P. (2014), "EEG Methods for the Psychological Sciences", Sage.

Ericsson, K. A. and Simon, H. A. (1993), "Protocol Analysis; Verbal Reports as Data", MIT Press.

Gero, J.S and Kannengiesser, U (2014), "The Function-Behaviour-Structure ontology of design", in Amaresh C. and Lucienne B. (eds), An Anthology of Theories and Models of Design, Springer, pp. 263-283. 
Goel, V. and Pirolli, P. (1992), “The structure of design problem spaces”, Cognitive Science Vol. 16, pp. 395-429.

Goel, V.and Vartanian, O. (2005), "Dissociating the roles of right ventral lateral and dorsal lateral prefrontal cortex in generating and maintenance of hypotheses in set-shift problems", Cerebral Cortex Vol. 15 No. 8 , pp. 1170-1177.

Goucher-Lambert, K., Moss J. and Cagan J. (2017), "Inside the mind: Using neuroimaging to understand moral product preference judgments involving sustainability", ASME Journal of Mechanical Design. Vol. 139 No. 4, pp. 041103-041103-11. https://dx.doi/org/10.1115/1.4035859.

Hinterberger, T., Zlabinger, M. and Blaser, K. (2014), "Neurophysiological correlates of various mental perspectives", Frontiers in Human Neuroscience, Vol. 8, pp. 1-16.

Kan, J. W. T., and Gero, J. S. (2017), "Quantitative Methods for Studying Design protocols”, Springer.

Kounios, J., and Beeman, M. (2014), “The Cognitive Neuroscience of Insight”, Annual Review of Psychology, Vol. 65, pp. 71-93.

Liang, C., Lin, C., Yao, C., Chang, W., Liu, Y. and Chen, S. (2017), "Visual attention and association: An electroencephalography study in expert designers", Design Studies, Vol. 48, pp. 76-95.

Liu, L., Nguyen, T., Zeng, Y. and Ben Hamza, A. (2016), "Identification of Relationships Between Electroencephalography (EEG) Bands and Design Activities", ASME 2016 International Design Engineering Technical Conferences and Computers and Information in Engineering Conference. Volume 7: 28th International Conference on Design Theory and Methodology. Charlotte, North Carolina, USA, August 21-24, 2016.

Liu, L., Li, Y., Xiong, Y., Cao, J. and Yuan, P. (2018), “An EEG study of the relationship between design problem statements and cognitive behaviors during conceptual design", Artificial Intelligence for Engineering Design, Analysis and Manufacturing Vol. 32, pp. 351-362.

Martindale, C. (1999), "Biological Bases of Creativity”, In Sternberg, R. (ed.), Handbook of Creativity, Cambridge University Press, Cambridge, pp. 137-151.

Nguyen, P., Nguyen, T., Zeng, Y. (2017), "Empirical approaches to quantifying effort, fatigue and concentration in the conceptual design process: an EEG study", Research in Engineering Design.

Pidgeon, L., Grealy, M., Duffy, A., Hay, L., McTeague, C., Vuletic, T., Coyle, D. and Gilbert, S. (2016), "Functional neuroimaging of visual creativity: a systematic review and meta-analysis", Brain and Behavior, Vol. 6 No. 10, pp. 1-26.

Pfurtscheller, G. and Lopes da Silva, F. (1999), "Event-related EEG/MEG synchronization and desynchronization: basic principles", Clin. Neurophysiol. Vol. 110, pp. 1842-1857.

Schön, D. (1988), “Designing: Rules, types and worlds”, Design Studies Vol. 9 No. 3, pp. 181-190.

Springer, S., Deutsch, G (1998), "Left brain, right brain (5th edition)", W.H. Freeman, San Francisco, CA.

Stern, R. and Ray, Q.K. (2001), "Psychophysical Recording”, Oxford University Press.

Van-Someren, M. W., Barnard, Y. F. and Sandberg, J. A. (1994), “The Think Aloud Method: A Practical Guide to Modelling Cognitive Processes", Academic Press.

Vecchiato, G., Jelic, A., Gaetano, T., Maglione, A., Matteis, F. and Babiloni, F. (2015), "Neurophysiological correlates of embodiment and motivational factors during the perception of virtual architectural", Conference Cognitive Processing, July.

Vergult, A., De Clercq, W., Palmini, A., Vanrumste, B., Dupont, P., Van Huffel, S., et al. (2007), "Improving the interpretation of ictal scalp eeg: BSS-cca algorithm for muscle artifact removal”, Epilepsia, Vol. 48, pp. 950-958.

Visser, W. (2009), "Design: one, but in different forms”, Design Studies, Vol. 30 No. 3, pp. 187-223.

Vos, D., Riès, S., Vanderperren, K., Vanrumste, B., Alario, F., Huffel, V. and Burle, B. (2010), "Removal of muscle artifacts from EEG recordings of spoken language production", Neuroinform, Vol. 8, pp. 135-150.

Ward, J. (2015), “The Student's Guide to Cognitive Neuroscience”, Psychology Press. 